\title{
Understanding the link between lipid diversity and the biophysical properties of the neuronal plasma membrane
}

\section{Supporting Information}

\author{
Katie A. Wilson, Hugo I. MacDermott-Opeskin, Eden Riley, Yiechang Lin, and Megan L. O'Mara* \\ Research School of Chemistry, College of Science, The Australian National University, Canberra, ACT, \\ 2601, Australia
}

*Corresponding author: megan.omara@anu.edu.au

\section{Table of Contents}

Expanded Methods

Table S1. Composition of the inner and outer leaflets for the membranes investigated in the current work.....S6

Table S2. Chemical composition of each of the lipids used in the present study.

Table S3. Available experimental biophysical properties of M1 to M8, as well as previously reported biophysical

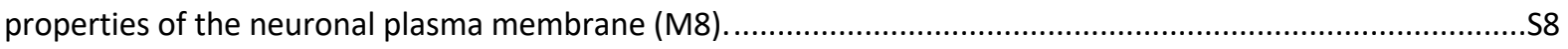

Table S4. Unsaturation per lipid for the membranes investigated in the current work.................................S8

Figure S1. Final snapshot of each of the membranes coloured by lipid class showing the extracellular and

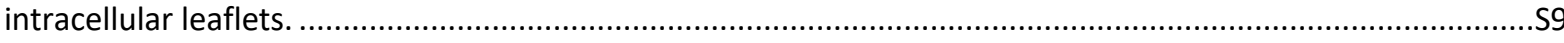

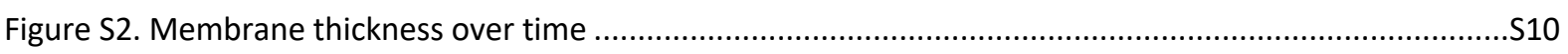

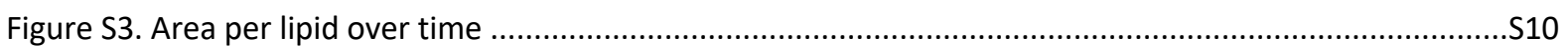

Figure S4. Average area per lipid for each of the unique lipid species .....................................................S11

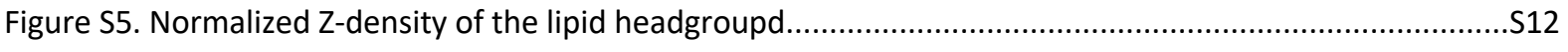

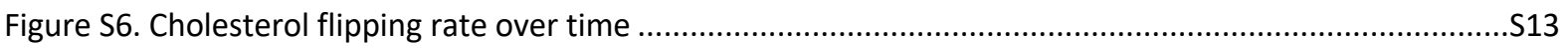

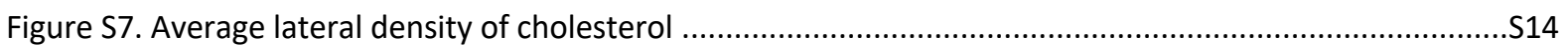

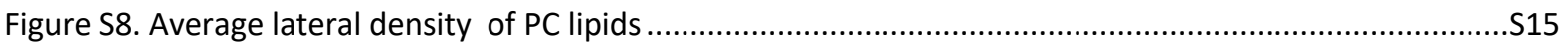

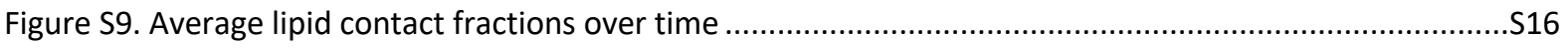

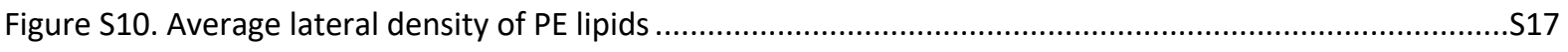

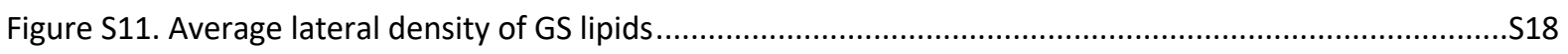

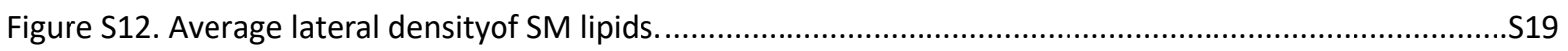

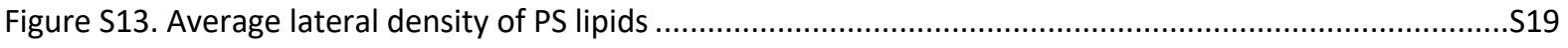

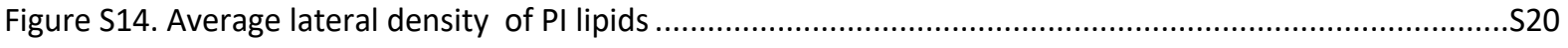

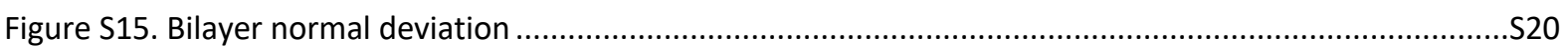

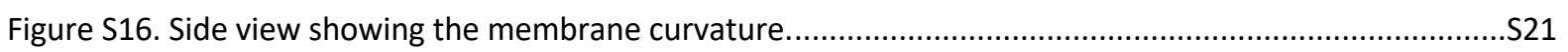




\section{Expanded Methods}

\section{Simulations}

The GROMACS 2018.3 molecular dynamics engine ${ }^{1-7}$ and the MARTINI 2.2P coarse grain forcefield parameters were used for all simulations. ${ }^{8}$ Lipid parameters from Ingólfsson et al. were used. ${ }^{9}$ Eight membranes, with compositions detailed in Table S1 were constructed using a custom version of the insane package. ${ }^{10}$ The simulation box size was $20 \times 20 \times 10 \mathrm{~nm}$ and periodic boundary conditions were applied in all directions. All systems employed the polarizable MARTINI water model ${ }^{11}$ along with a $150 \mathrm{mM}$ sodium chloride concentration.

Each membrane was energy minimized using the steepest descent algorithm and equilibrated prior to production simulation. Equilibration was conducted for $50 \mathrm{~ns}$ with a $10 \mathrm{fs}$ time step at constant temperature and pressure. A temperature of $310 \mathrm{~K}$ was maintained using the Bussi thermostat ${ }^{12}$ with a coupling constant of $1.0 \mathrm{ps}$. The pressure was maintained at 1 bar using a semi-isotropic Berendsen barostat with a compressibility of $3 \times 10^{-4} \mathrm{bar}^{-1}$ and a coupling constant of $12 \mathrm{ps}$. Following equilibration, production simulations were conducted for $30 \mu \mathrm{s}$ in triplicate at constant temperature and pressure. Each replicate was initialized from a different set of starting velocities. The temperature was maintained as described above, whilst semi-isotropic Parrinello-Rahman pressure coupling ${ }^{13,14}$ was used to maintain a pressure of 1 bar with a compressibility of $3 \times 10^{-4}$ bar $^{-1}$ and a coupling constant of 12 ps. All simulations were conducted according to the recommended standard MARTINI configuration, ${ }^{15}$ with a timestep of $20 \mathrm{fs}$.

Each membrane was analysed and compared in terms of membrane biophysical properties. The ROH, PO4 and C3 beads were used as reference for cholesterol, phospholipids and sphingolipids, respectively. Reported averages over the total simulation time include standard error of the mean (SEM) between replicates.

\section{Thickness and Area Per Lipid}

Membrane thickness was computed using FATSLiM. ${ }^{16}$ Area per lipid was computed using FATSLiM ${ }^{16}$ and the MemSurfer package employing the smooth membrane kernel, with a kernel width of $10 \mathrm{~nm} .{ }^{17}$

\section{Lipid flipflop}

The propensity of lipids in each membrane to flipflop between leaflets was assessed using a python analysis tool, employing the FATSLiM analysis package ${ }^{16}$ and MDTraj 1.9.3 library. ${ }^{18}$ As weak position restraints to suppress bilayer undulations were not employed, a different approach to that of Ingólfsson et al was used. ${ }^{9}$ Firstly, a large group of non-flipping reference lipids was selected to enable the calculation of local bilayer thicknesses and headgroup positioning. The FATSLiM membrane identifier was then used to assign groups of non-flipping reference lipids to each leaflet based on the position of their reference beads. Following this, distances in the $x-y$ plane between each flip-flopping lipid's reference bead and its $\mathrm{N}$ nearest-neighbour non-flipping lipids was calculated. The $\mathrm{z}-$ coordinates of the non-flipping lipids were averaged over the $\mathrm{N}$ nearest neighbours in each leaflet to provide a local approximation to the thickness and headgroup positioning around each flipping molecule. Convergence with respect to the number of nearest neighbours was assessed and $\mathrm{N}=5-10$ determined to provide good performance. We note that as $\mathrm{N}$ increases, convergence to a global average as is achieved. Following calculation of local thicknesses and headgroup positioning around each flip-flopping lipid, local regions defining the upper and lower leaflet were assessed using a cutoff of $1.1 \mathrm{~nm}$ in the z-direction. From this each flip-flopping lipid can be assigned to the upper leaflet, the lower leaflet or interstitial region. Definition of leaflet regions then allows transitions between the upper and lower leaflets to be identified. Time dependent flipping rates were evaluated using 100 ns blocks. 


\section{Densities}

Z-axis density of lipid classes was calculated using the GROMACS tool gmx_density based on the reference headgroup beads. Z-density values were then normalized by the maximum density value. The number density of lipid classes in the $x-y$ plane was assessed using the g_mydensity tool and visualized using the matplotlib library. ${ }^{19}$ The colour scale for each density map was proportional to the number of lipids of each class present in the membrane.

\section{Contact fractions}

Propensity of lipid species and lipid classes to collocate was assessed using lipid contact fractions, as has been previously reported in the literature..$^{20}$ Firstly, each lipid species is assigned a reference bead and the neighbouring reference beads within $1.2 \mathrm{~nm}$ ( $1.1 \mathrm{for} \mathrm{CHOL}$ ) assessed. The relative enrichment and depletion of each species $B$ around the primary lipid $A$ is then calculated by comparing the local molar ratio within the $1.2 \mathrm{~nm}$ cut-off ( $1.1 \mathrm{~nm}$ for $\mathrm{CHOL}$ ) to the global molar ratio of the primary lipid. These values are then averaged over all lipid species within the lipid class. Contact fractions can then be averaged over time, resulting in the expression below where the subscript $t, m o l$ indicates that the ensemble average runs over both molecules included in a class and over time.

$$
C=\left\langle\frac{B_{A}}{B_{\text {total }}}\right\rangle_{t, \text { mol }}
$$

Where $B_{A}$ is the molar fraction of $B$ in the environment of the primary lipid $A$ and $B_{\text {total }}$ is the molar fraction of $B$ in the whole system. We note that in this work, contact fractions have been evaluated over the entire membrane rather than split by leaflet, resulting in enhanced contact fraction values for lipids that only occur in one leaflet. Additionally, contact ratios observed in our study are more extreme than those of the large patch neuronal plasma membrane, ${ }^{9}$ likely due to a smaller system size which will magnify the effect of domain formation on contact ratios.

\section{Self-diffusivities}

Diffusion of lipids in the $x$-y plane was assessed by computation of lateral self-diffusivities from the Mean Squared Displacement (MSD) of each lipid class. The 200 to 500 ns lag-time portion of the MSD was used to avoid ballistic trajectories obtained at short lag-times and poor averaging obtained at long lag-times. Following the recommendations of a recent review, ${ }^{21}$ log-log plots of the MSD against lagtime were used to confirm that the 200 to 500 ns lag time portion of the MSD was the appropriate segment for linear fitting to obtain lateral self-diffusivities. Due to the need to employ contiguous trajectories, the final $10 \mu \mathrm{s}$ of each replicate for each membrane was used and lateral self-diffusivities averaged over the three replicates.

\section{Membrane curvature}

Curvature of the membranes was assessed using bilayer normal deviations. Oriented normals from MemSurfer were compared to the global bilayer normal ( $z$ axis) and the angle between these two vectors calculated. These values are then averaged over all lipids and fit to a smooth histogram employing a kernel density estimate.

\section{References:}

(1) Abraham, M. J.; Murtola, T.; Schulz, R.; Páll, S.; Smith, J. C.; Hess, B.; Lindahl, E. GROMACS: High performance molecular simulations through multi-level parallelism from laptops to supercomputers. SoftwareX 2015, 1, 19.

(2) Páll, S.; Abraham, M. J.; Kutzner, C.; Hess, B.; Lindahl, E. International Conference on Exascale Applications and Software, 2014; $p 3$. 
(3) Pronk, S.; Páll, S.; Schulz, R.; Larsson, P.; Bjelkmar, P.; Apostolov, R.; Shirts, M. R.; Smith, J. C.; Kasson, P. M.; van der Spoel, D. GROMACS 4.5: a high-throughput and highly parallel open source molecular simulation toolkit. Bioinformatics 2013, 29 (7), 845.

(4) Hess, B.; Kutzner, C.; Van Der Spoel, D.; Lindahl, E. GROMACS 4: algorithms for highly efficient, load-balanced, and scalable molecular simulation. J. Chem. Theory Comput. 2008, 4 (3), 435.

(5) Van Der Spoel, D.; Lindahl, E.; Hess, B.; Groenhof, G.; Mark, A. E.; Berendsen, H. J. GROMACS: fast, flexible, and free. J. Comput. Chem. 2005, 26 (16), 1701.

(6) Lindahl, E.; Hess, B.; Van Der Spoel, D. GROMACS 3.0: a package for molecular simulation and trajectory analysis. Molecular modeling annual 2001, 7 (8), 306.

(7) Berendsen, H. J.; van der Spoel, D.; van Drunen, R. GROMACS: a message-passing parallel molecular dynamics implementation. Comput. Phys. Commun. 1995, 91 (13), 43.

(8) de Jong, D. H.; Singh, G.; Bennett, W. F. D.; Arnarez, C.; Wassenaar, T. A.; Schäfer, L. V.; Periole, X.; Tieleman, D. P.; Marrink, S. J. Improved Parameters for the Martini Coarse-Grained Protein Force Field. J. Chem. Theory Comput. 2013, 9 (1), 687.

(9) Ingólfsson, H. I.; Carpenter, T. S.; Bhatia, H.; Bremer, P.-T.; Marrink, S. J.; Lightstone, F. C. Computational Lipidomics of the Neuronal Plasma Membrane. Biophys. J. 2017, 113 (10), 2271.

(10) Wassenaar, T. A.; Ingólfsson, H. I.; Böckmann, R. A.; Tieleman, D. P.; Marrink, S. J. Computational Lipidomics with insane: A Versatile Tool for Generating Custom Membranes for Molecular Simulations. J. Chem. Theory Comput. 2015, 11 (5), 2144.

(11) Yesylevskyy, S. O.; Schäfer, L. V.; Sengupta, D.; Marrink, S. J. Polarizable water model for the coarse-grained MARTINI force field. PLoS Comp. Biol. 2010, 6 (6).

(12) Bussi, G.; Donadio, D.; Parrinello, M. Canonical sampling through velocity rescaling. The Journal of chemical physics 2007, 126 (1), 014101.

(13) Parrinello, M.; Rahman, A. Crystal structure and pair potentials: A moleculardynamics study. Phys. Rev. Lett. 1980, 45 (14), 1196.

(14) Parrinello, M.; Rahman, A. ACS Catalysis. J. Appl. Phys. 1981, 52 (12), 7182.

(15) de Jong, D. H.; Baoukina, S.; Ingólfsson, H. I.; Marrink, S. J. Martini straight: Boosting performance using a shorter cutoff and GPUs. Comput. Phys. Commun. 2016, 199, 1.

(16) Buchoux, S. FATSLiM: a fast and robust software to analyze MD simulations of membranes. Bioinformatics 2017, 33 (1), 133.

(17) Bhatia, H.; Ingólfsson, H. I.; Carpenter, T. S.; Lightstone, F. C.; Bremer, P.-T. MemSurfer: A Tool for Robust Computation and Characterization of Curved Membranes. J. Chem. Theory Comput. 2019, 15 (11), 6411.

(18) McGibbon, Robert T.; Beauchamp, Kyle A.; Harrigan, Matthew P.; Klein, C.; Swails, Jason M.; Hernández, Carlos X.; Schwantes, Christian R.; Wang, L.-P.; Lane, Thomas J.; Pande, Vijay S. MDTraj: A Modern Open Library for the Analysis of Molecular Dynamics Trajectories. Biophys. J. 2015, 109 (8), 1528.

(19) Castillo, N.; Monticelli, L.; Barnoud, J.; Tieleman, D. P. Free energy of WALP23 dimer association in DMPC, DPPC, and DOPC bilayers. Chem. Phys. Lipids 2013, 169, 95.

(20) Kolds $\varnothing$, H.; Shorthouse, D.; Hélie, J.; Sansom, M. S. P. Lipid Clustering Correlates with Membrane Curvature as Revealed by Molecular Simulations of Complex Lipid Bilayers. PLoS Comp. Biol. 2014, 10 (10), e1003911. 
(21) Maginn, E. J.; Messerly, R. A.; Carlson, D. J.; Roe, D. R.; Elliott, J. R. Best practices for computing transport properties 1 . self-diffusivity and viscosity from equilibrium molecular dynamics [article v1. 0]. Living J. Comput. Mol. Sci 2019, 1, 1. 
Table S1. Composition of the inner and outer leaflets for the membranes investigated in the current work with the percent composition and number of lipids (brackets) of each species listed. ${ }^{a}$

\begin{tabular}{|c|c|c|c|c|c|c|c|c|c|c|c|c|c|c|c|c|}
\hline & \multicolumn{2}{|c|}{ Membrane 1} & \multicolumn{2}{|c|}{ Membrane 2} & \multicolumn{2}{|c|}{ Membrane 3} & \multicolumn{2}{|c|}{ Membrane 4} & \multicolumn{2}{|c|}{ Membrane 5} & \multicolumn{2}{|c|}{ Membrane 6} & \multicolumn{2}{|c|}{ Membrane 7} & \multicolumn{2}{|c|}{ Membrane 8} \\
\hline & Inner & Outer & Inner & Outer & Inner & Outer & Inner & Outer & Inner & Outer & Inner & Outer & Inner & Outer & Inner & Outer \\
\hline $\mathrm{CHOL}$ & $20 \%(135)$ & $20 \%(135)$ & $44.6 \%(301)$ & $44.4 \%(300)$ & $44.6 \%(301)$ & $44.4 \%(300)$ & $44.7 \%(301)$ & $44.6 \%(300)$ & $44.8 \%(301)$ & $44.8 \%(300)$ & $44.7 \%(301)$ & $44.6 \%(300)$ & $44.9 \%(301)$ & $44.7 \%(300)$ & $45.8 \%(301)$ & $45.6 \%(299)$ \\
\hline POPC & $80 \%(540)$ & $80 \%(540)$ & $55.4 \%$ (374) & $55.6 \%(375)$ & $22.2 \%(150)$ & $38.1 \%(257)$ & $15.9 \%(107)$ & $26.2 \%(176)$ & $8.0 \%(54)$ & $13.9 \%(93)$ & $22.3 \%(150)$ & $38.2 \%(257)$ & $8.1 \%(54)$ & $13.9 \%(93)$ & $9.3 \%(33)$ & $8.8 \%(58)$ \\
\hline PUPE & & & & & $33.2 \%(224)$ & $17.5 \%(118)$ & $25.1 \%$ (169) & $11.7 \%(79)$ & $33.3 \%(224)$ & $17.6 \%$ (118) & $15.2 \%(102)$ & $8.0 \%(54)$ & $15.2 \%(102)$ & $8.0 \%(54)$ & $18.3 \%(65)$ & $5.0 \%(33)$ \\
\hline DPSM & - & - & - & - & - & - & $2.7 \%(18)$ & $9.5 \%(64)$ & - & - & - & - & - & - & $2.8 \%(10)$ & $5.9 \%(39)$ \\
\hline PAPS & & & & & & & $11.7 \%(79)$ & $0 \%(0)$ & & & & & & & $5.1 \%(18)$ & $0 \%(0)$ \\
\hline DPGS & & & & & & & $0 \%(0)$ & $8.0 \%(54)$ & & & & & & & $0 \%(0)$ & $5.0 \%(33)$ \\
\hline DPPC & & - & & & & & & & $4.9 \%(33)$ & $8.4 \%(56)$ & - & & $4.9 \%(33)$ & $8.3 \%(56)$ & $5.6 \%(20)$ & $5.3 \%(35)$ \\
\hline PAPC & & - & & & & & & & $4.3 \%(29)$ & $6.9 \%(46)$ & & & $4.3 \%(29)$ & $7.3 \%(49)$ & $4.8 \%(17)$ & $4.7 \%(31)$ \\
\hline DOPC & - & & - & - & - & & - & & $1.9 \%(13)$ & $3.4 \%(23)$ & & - & $1.9 \%(13)$ & $3.4 \%(23)$ & $2.2 \%(8)$ & $2.1 \%(14)$ \\
\hline PUPC & & - & & & & & & & $1.5 \%(10)$ & $2.7 \%(18)$ & & & $1.5 \%(10)$ & $2.7 \%(18)$ & $1.7 \%(6)$ & $1.7 \%(11)$ \\
\hline OIPC & - & - & - & - & & & - & & $0.4 \%(3)$ & $0.9 \%(6)$ & - & - & $0.4 \%(3)$ & $0.9 \%(6)$ & $0.6 \%(2)$ & $0.6 \%(4)$ \\
\hline PFPC & - & - & - & - & & & & & $4 \%(3)$ & $0.9 \%(6)$ & & - & $0.4 \%(3)$ & $0.9 \%(6)$ & $0.6 \%(2)$ & $0.6 \%(4)$ \\
\hline OUPC & - & - & - & - & - & & - & & $0.3 \%(2)$ & $0.6 \%(4)$ & & - & $0.3 \%(2)$ & $0.6 \%(4)$ & $0.3 \%(1)$ & $0.3 \%(2)$ \\
\hline PAPE & & & & & & & & & & & $9.5 \%(64)$ & $4.9 \%(33)$ & $9.6 \%(64)$ & $4.9 \%(33)$ & $11.5 \%(41)$ & $3.0 \%(20)$ \\
\hline POPE & & & & & & & & & & & $3.9 \%(26)$ & $2.1 \%(14)$ & $3.9 \%(26)$ & $2.1 \%(14)$ & $4.5 \%(16)$ & $1.2 \%(8)$ \\
\hline OUPE & & & & & & & & & & & $2.1 \%(14)$ & $1.0 \%(7)$ & $2.1 \%$ (14) & $1.0 \%(7)$ & $2.5 \%(9)$ & $0.6 \%(4)$ \\
\hline OAPE & & & & & & & & & & & $1.9 \%(13)$ & $1.0 \%(7)$ & $1.9 \%(13)$ & $1.0 \%(7)$ & $2.2 \%(8)$ & $0.6 \%(4)$ \\
\hline OIPE & & & & & & & & & & & $0.4 \%(3)$ & $0.1 \%(1)$ & $0.4 \%(3)$ & $0.1 \%(1)$ & $0.6 \%(2)$ & $0 \%(0)$ \\
\hline PNSM & - & - & - & - & - & - & - & - & - & - & - & - & - & - & $0.6 \%(2)$ & $1.2 \%(8)$ \\
\hline PBSM & - & - & - & - & - & - & - & - & - & - & - & - & - & - & $0.6 \%(2)$ & $1.1 \%(7)$ \\
\hline POSM & - & - & - & - & - & - & - & - & - & - & - & - & - & - & $0.3 \%(1)$ & $0.6 \%(4)$ \\
\hline PUPS & & & & & & & & & & & & & & & $6.2 \%(22)$ & $0 \%(0)$ \\
\hline POPS & & & & & & & & & & & & & & & $4.5 \%(16)$ & $0 \%(0)$ \\
\hline OUPS & & & & & & & & & & & & & & & $1.1 \%(4)$ & $0 \%(0)$ \\
\hline DPPS & & & & & & & & & & & & & & & $0.8 \%(3)$ & $0 \%(0)$ \\
\hline PNGS & & & & & & & & & & & & & & & $0 \%(0)$ & $1.1 \%(7)$ \\
\hline DBGS & & & & & & & & & & & & & & & $0 \%(0)$ & $0.9 \%(6)$ \\
\hline DPG1 & & & & & & & & & & & & & & & $0 \%(0)$ & $0.9 \%(6)$ \\
\hline DPG3 & & & & & & & & & & & & & & & $0 \%(0)$ & $0.9 \%(6)$ \\
\hline POGS & & & & & & & & & & & & & & & $0 \%(0)$ & $0.6 \%(4)$ \\
\hline DBG1 & & & & & & & & & & & & & & & $0 \%(0)$ & $0.2 \%(1)$ \\
\hline DBG3 & & & & & & & & & & & & & & & $0 \%(0)$ & $0.2 \%(1)$ \\
\hline PNG1 & & & & & & & & & & & & & & & $0 \%(0)$ & $0.2 \%(1)$ \\
\hline PNG3 & & & & & & & & & & & & & & & $0 \%(0)$ & $0.2 \%(1)$ \\
\hline PUPI & & - & & & & & & & & & & & - & & $3.7 \%(13)$ & $0 \%(0)$ \\
\hline POPI & & & & & - & - & - & - & - & & - & & & & $2.2 \%(8)$ & $0 \%(0)$ \\
\hline PAPI & & - & & & & & & & & & & & - & & $2.2 \%(8)$ & $0 \%(0)$ \\
\hline PIPI & - & & & - & - & & - & & - & - & - & - & & & $0.8 \%(3)$ & $0 \%(0)$ \\
\hline PAP1 & & - & & & & & & & & & & & - & & $0.6 \%(2)$ & $0 \%(0)$ \\
\hline PAP2 & - & & & - & - & & - & & & - & & & & & $0.6 \%(2)$ & $0 \%(0)$ \\
\hline PAP3 & & - & & & & & & & & & & & - & & $0.6 \%(2)$ & $0 \%(0)$ \\
\hline POP1 & & & & & & & & & & & & & & & $0.3 \%(1)$ & $0 \%(0)$ \\
\hline POP2 & & - & & & & & & & & & & & - & & $0.3 \%(1)$ & $0 \%(0)$ \\
\hline POP3 & & & & & & & & & & & & & & & $0.3 \%(1)$ & $0 \%(0)$ \\
\hline PADG & & & & & & & & & & & & & & & $0.6 \%(2)$ & $0.3 \%(2)$ \\
\hline DPCE & & - & & & & & & & & & & - & - & & $0.6 \%(2)$ & $0.3 \%(2)$ \\
\hline PAPA & & & & & & & & & & & & & & & $0.6 \%(2)$ & $0 \%(0)$ \\
\hline PPC & & - & & & & & & & & & & - & - & - & $0 \%(0)$ & $0.2 \%(1)$ \\
\hline IPE & - & & & - & - & - & - & . & . & - & - & - & - & - & $0.3 \%(1)$ & $0 \%(0)$ \\
\hline
\end{tabular}


Table S2. Chemical composition of each of the lipids used in the present study. ${ }^{a}$

\begin{tabular}{|c|c|c|c|c|}
\hline & Lipid Class & sn1 tailb & sn2 tailb & Total unsaturation \\
\hline POPC & $\mathrm{PC}$ & $\mathrm{CDCC}$ & $\mathrm{CCCC}$ & 1 \\
\hline PUPE & $\mathrm{PE}$ & DDDDD & $\mathrm{CCCC}$ & 5 \\
\hline DPSM & SM & TCC & CCCC & 1 \\
\hline PAPS & PS & DDDDC & CCCC & 4 \\
\hline DPGS & GS & TCCC & CCCC & 1 \\
\hline DPPC & PC & $\mathrm{CcCC}$ & $\mathrm{CCCC}$ & 0 \\
\hline PAPC & PC & DDDDC & $\mathrm{CCCC}$ & 4 \\
\hline DOPC & PC & $\mathrm{CDCC}$ & $\mathrm{CDCC}$ & 2 \\
\hline PUPC & PC & DDDDD & $\mathrm{CCCC}$ & 5 \\
\hline OIPC & PC & $\mathrm{CDDC}$ & $\mathrm{CDCC}$ & 3 \\
\hline PFPC & PC & CDDD & $\mathrm{CCCC}$ & 3 \\
\hline OUPC & PC & DDDDD & $\mathrm{CDCC}$ & 6 \\
\hline PAPE & $\mathrm{PE}$ & DDDDC & $\mathrm{CCCC}$ & 4 \\
\hline POPE & $\mathrm{PE}$ & $\mathrm{CDCC}$ & $\mathrm{CCCC}$ & 1 \\
\hline OUPE & $\mathrm{PE}$ & DDDDD & $\mathrm{CDCC}$ & 6 \\
\hline OAPE & PE & DDDDC & $\mathrm{CDCC}$ & 5 \\
\hline OIPE & $\mathrm{PE}$ & $\mathrm{CDDC}$ & $\mathrm{CDCC}$ & 3 \\
\hline PNSM & SM & TCC & CCCDCC & 2 \\
\hline PBSM & SM & TCC & ccccc & 1 \\
\hline POSM & SM & TCC & $\mathrm{CDCC}$ & 2 \\
\hline PUPS & PS & DDDDD & $\mathrm{CCCC}$ & 5 \\
\hline POPS & PS & $\mathrm{CDCC}$ & $\mathrm{CCCC}$ & 1 \\
\hline OUPS & PS & DDDDD & $\mathrm{CDCC}$ & 6 \\
\hline DPPS & PS & $\mathrm{cCCC}$ & $\mathrm{CCCC}$ & 0 \\
\hline PNGS & GS & TCCC & CCCDCC & 2 \\
\hline DBGS & GS & TCCCC & $\mathrm{CcCCC}$ & 1 \\
\hline DPG1 & GS & TCC & $\mathrm{CCCC}$ & 1 \\
\hline DPG3 & GS & TCC & $\mathrm{CCCC}$ & 1 \\
\hline POGS & GS & TCCC & $\mathrm{CDCC}$ & 2 \\
\hline DBG1 & GS & TCCC & $\mathrm{CcCCC}$ & 1 \\
\hline DBG3 & GS & TCCC & CCCCC & 1 \\
\hline PNG1 & GS & TCC & CCCDCC & 2 \\
\hline PNG3 & GS & TCC & CCCDCC & 2 \\
\hline PUPI & $\mathrm{PI}$ & DDDDD & CCCC & 5 \\
\hline POPI & $\mathrm{PI}$ & $\mathrm{CDCC}$ & $\mathrm{CCCC}$ & 1 \\
\hline PAPI & $\mathrm{PI}$ & DDDDC & $\mathrm{CCCC}$ & 4 \\
\hline PIPI & $\mathrm{PI}$ & $\mathrm{CDDC}$ & $\mathrm{CCCC}$ & 2 \\
\hline PAP1 & $\mathrm{PI}$ & DDDDC & $\mathrm{CCCC}$ & 4 \\
\hline PAP2 & PI & DDDDC & $\mathrm{CCCC}$ & 4 \\
\hline PAP3 & $\mathrm{PI}$ & DDDDC & $\mathrm{CCCC}$ & 4 \\
\hline POP1 & $\mathrm{PI}$ & $\mathrm{CDCC}$ & $\mathrm{CCCC}$ & 1 \\
\hline POP2 & $\mathrm{PI}$ & CDCC & $\mathrm{CCCC}$ & 1 \\
\hline POP3 & PI & $\mathrm{CDCC}$ & $\mathrm{CCCC}$ & 1 \\
\hline PADG & DG & DDDDC & $\mathrm{CCCC}$ & 4 \\
\hline DPCE & $\mathrm{CE}$ & TCC & $\mathrm{CCCC}$ & 1 \\
\hline PAPA & PA & DDDDC & CCCC & 4 \\
\hline PPC & Lyso-PC & $\mathrm{CCCC}$ & - & 0 \\
\hline IPE & Lyso-PE & $\mathrm{CDDC}$ & - & 2 \\
\hline
\end{tabular}

a Lipids are coloured based on classification as PC (green), PE (orange), SM (red), PS (purple), GS (yellow) PI (blue) and other (gray). ${ }^{\mathrm{b}}$ Tail saturation listed though MARTINI beads, where $C$ represents a saturated carbon, D represents a cis unsaturation and T represents a trans unsaturation. 
Table S3. Available experimental biophysical properties of M1 to M8, as well as previously reported biophysical properties of the neuronal plasma membrane (M8).

\begin{tabular}{|c|c|c|c|c|}
\hline Composition & Property & Value & Temperature $(\mathrm{K})$ & Method \\
\hline $100 \% \mathrm{POPC}^{\mathrm{a}}$ & Thickness $\left(D_{H H}\right)$ & $36.5 \AA$ & 303 & SAXS/SANS \\
\hline $100 \%$ POPC $^{b}$ & Thickness (Dнн) & $36.7 \pm 0.2 \AA$ & 303 & SAXS/SANS \\
\hline $20 \% \mathrm{CHOL} 80 \%$ POPC (M1)c & APL & $53 A^{2}$ & 297 & Langmuir film balance \\
\hline $13 \%$ CHOL $87 \%$ POPC ( M1) & $D_{x y}$ & $1.29 \times 10^{-7} \mathrm{~cm}^{2} \mathrm{~s}$ & 313 & pfg- ${ }^{1} \mathrm{H} N M R$ \\
\hline $50 \%$ CHOL 50\% POPC ( M2) & APL & $45.1 \pm 0.9 \AA^{2}$ & 321 & Local field ${ }^{13} \mathrm{C}$ NMR \\
\hline $40 \%$ CHOL $60 \%$ POPC ( M2) & APL & $46 \AA^{2}$ & 297 & Langmuir film balance \\
\hline $48 \%$ CHOL 52\% POPC ( M2) $)^{d}$ & $D_{x y}$ & $7 \times 10^{-6} \mathrm{~cm}^{2} \mathrm{~s}$ & 313 & pfg- ${ }^{1} \mathrm{H} N M R$ \\
\hline $50 \% \mathrm{CHOL} 50 \%$ POPC $(\sim \mathrm{M} 2)^{\mathrm{e}}$ & Thickness $\left(D_{B^{\prime}}\right)$ & $57.8 \pm 3.5 \AA$ & 321 & Local field ${ }^{13} \mathrm{C}$ NMR \\
\hline Neuronal $(\mathrm{M} 8)^{\mathrm{f}}$ & Thickness $\left(D_{H н}\right)$ & $40.575 \pm 0.02 \AA$ & 310 & MD \\
\hline Neuronal $(\mathrm{M} 8)^{f}$ & APL (outer) & $46.0 \AA^{2}$ & 310 & MD \\
\hline Neuronal $(\mathrm{M} 8)^{f}$ & APL (inner) & $48.5 \AA^{2}$ & 310 & MD \\
\hline Neuronal $(M 8)^{f}$ & $\mathrm{D}_{\mathrm{xy}}$ (inner) & $2.85 \pm 0.2 \times 10^{-7} \mathrm{~cm}^{2} \mathrm{~s}$ & 310 & MD \\
\hline Neuronal $(M 8)^{f}$ & $D_{x y}$ (outer) & $1.65 \pm 0.2 \times 10^{-7} \mathrm{~cm}^{2} \mathrm{~s}$ & 310 & MD \\
\hline Neuronal $(\mathrm{M} 8)^{\mathrm{f}}$ & CHOL flip-flop & $4.8205 \pm 0.004 \times 10^{6} \mathrm{~s}^{-1}$ & 310 & $\mathrm{MD}$ \\
\hline
\end{tabular}

aKučerka, N.; Nieh, M.-P.; Katsaras, J. Fluid phase lipid areas and bilayer thicknesses of commonly used phosphatidylcholines as a function of temperature. Biochim. Biophys. Acta 2011, 1808 (11), 2761. bFogarty, J. C.; Arjunwadkar, M.; Pandit, S. A.; Pan, J. Atomically detailed lipid bilayer models for the interpretation of small angle neutron and X-ray scattering data. Biochim. Biophys. Acta 2015, 1848 (2), 662.'Smaby, J. M.; Momsen, M. M.; Brockman, H. L.; Brown, R. E. Phosphatidylcholine acyl unsaturation modulates the decrease in interfacial elasticity induced by cholesterol. Biophys. J. 1997,73 (3), 1492. 'FFilippov, A.; Orädd, G.; Lindblom, G. Influence of Cholesterol and Water Content on Phospholipid Lateral Diffusion in Bilayers. Langmuir 2003,19 (16), 6397. eLeftin, A.; Molugu, Trivikram R.; Job, C.; Beyer, K.; Brown, Michael F. Area per Lipid and Cholesterol Interactions in Membranes from Separated Local-Field 13C NMR Spectroscopy. Biophys. J. 2014, 107 (10), 2274. fIngólfsson, H. I.; Carpenter, T. S.; Bhatia, H.; Bremer, P.-T.; Marrink, S. J.; Lightstone, F. C. Computational Lipidomics of the Neuronal Plasma Membrane. Biophys. J. 2017, 113 (10), 2271.

Table S4. Number of unsaturation per lipid for the membranes investigated in the current work. ${ }^{\mathrm{a}}$

\begin{tabular}{lccc}
\hline & Inner Leaflet & Outer Leaflet & Overall Membrane \\
\hline M1 & 1.0 & 1.0 & 1.0 \\
M2 & 1.0 & 1.0 & 1.0 \\
M3 & 3.4 & 2.3 & 2.8 \\
M4 & 3.4 & 1.8 & 2.6 \\
M5 & 3.8 & 2.9 & 3.3 \\
M6 & 3.0 & 2.0 & 2.5 \\
M7 & 3.3 & 2.6 & 3.0 \\
M8 & 3.3 & 2.1 & 2.7 \\
\hline
\end{tabular}




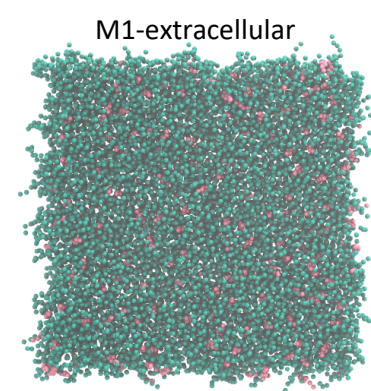

M2-extracellular

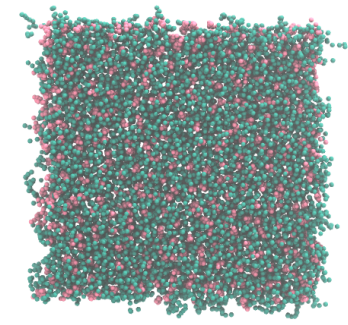

M3-extracellular

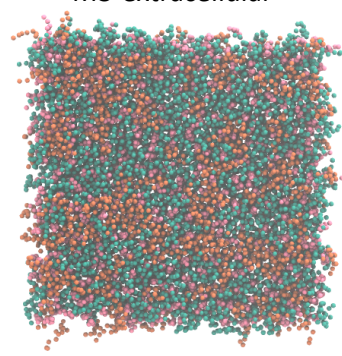

M4-extracellular

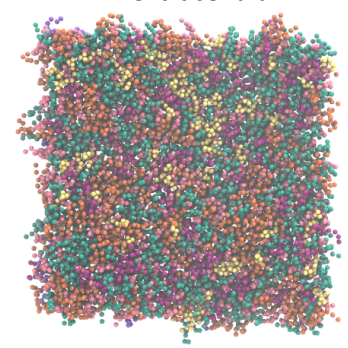

M1-intracellular

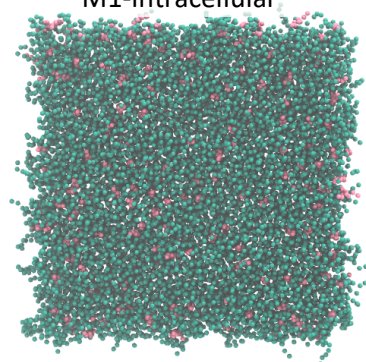

M2-intracellular

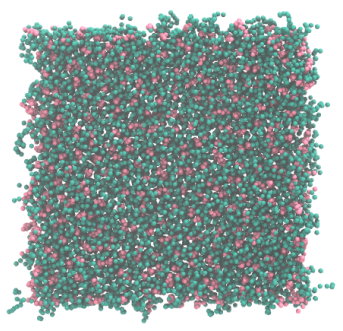

M3-intracellular

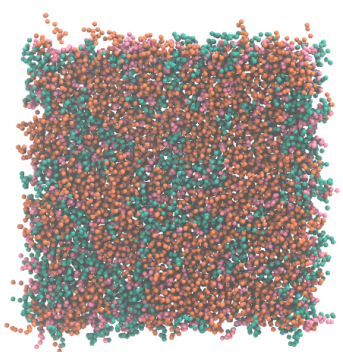

M4-intracellular

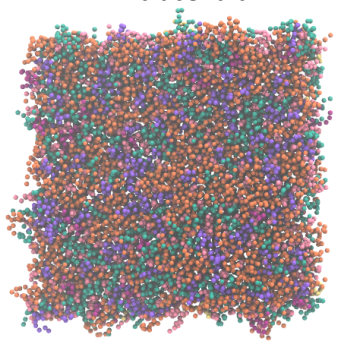

$\backsim \mathrm{CHOL} \backsim \mathrm{PC} \backsim \mathrm{PE} \backsim \mathrm{SM} \backsim \mathrm{PS} \backsim \mathrm{GS} \backsim \mathrm{PI} \backsim$ Other

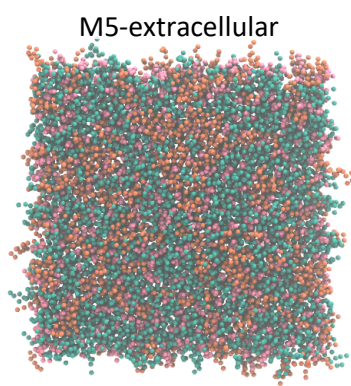

M6-extracellular

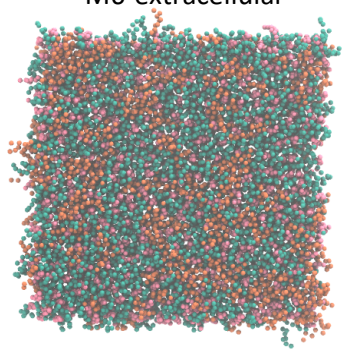

M7-extracellular

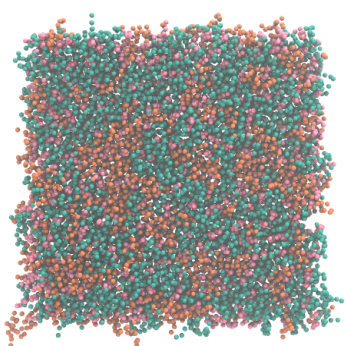

M8-extracellular

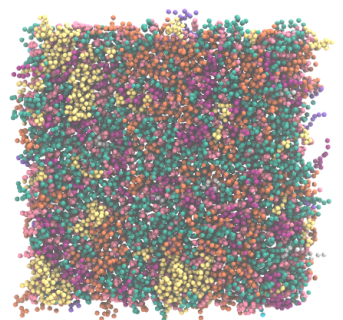

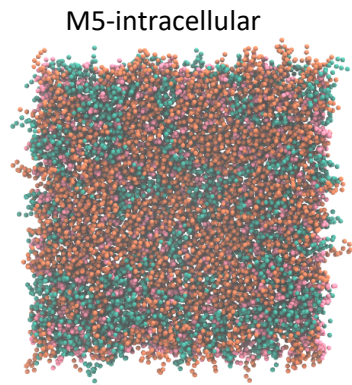

M6-intracellular

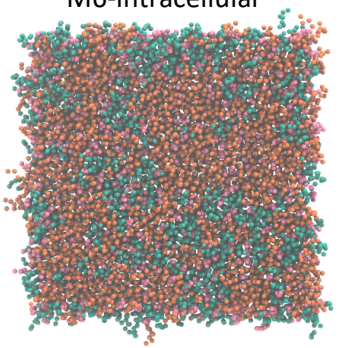

M7-intracellular

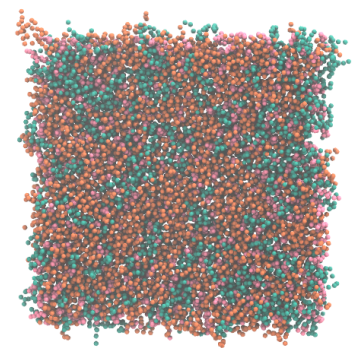

M8-intracellular

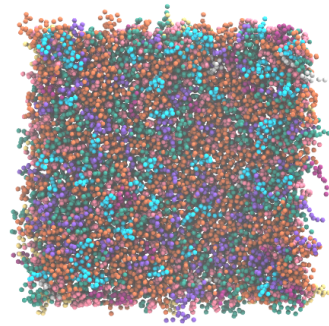

Figure S1. Final snapshot of each of the membranes coloured by lipid class showing the extracellular and intracellular leaflets. 


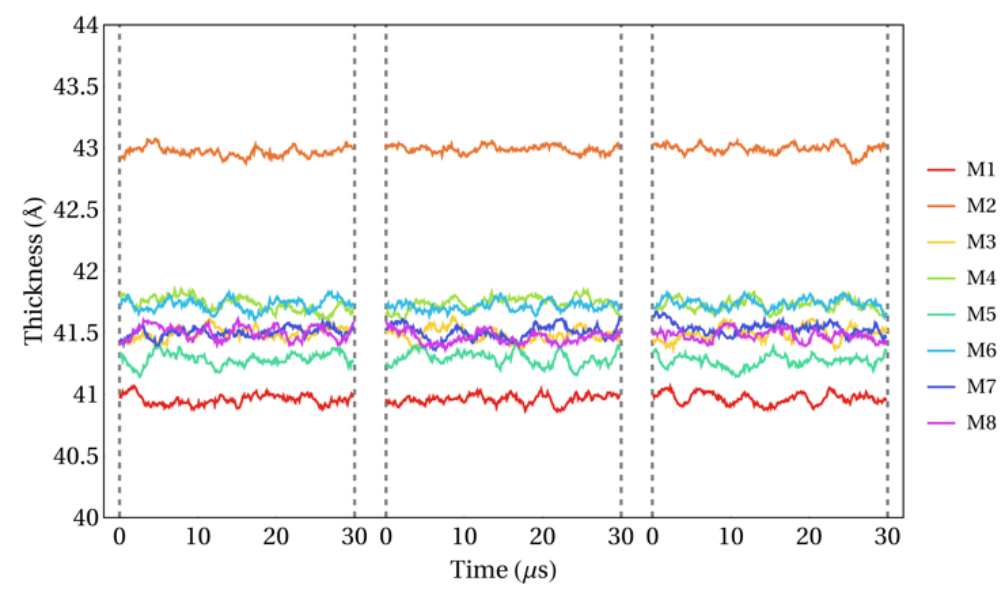

Figure S2. Membrane thickness over time for replica 1 (left), 2 (middle) and 3 (right) in each of the 8 membranes modelled. Timeseries were smoothed using a running average filter. See Figure 1 and Table S1 for composition of each membrane.

a)

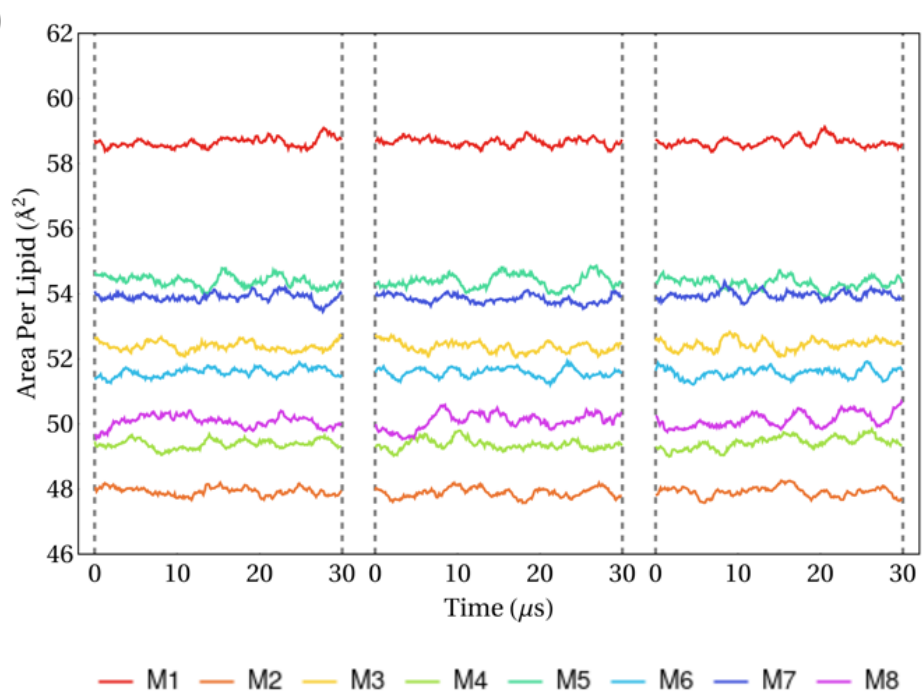

b)

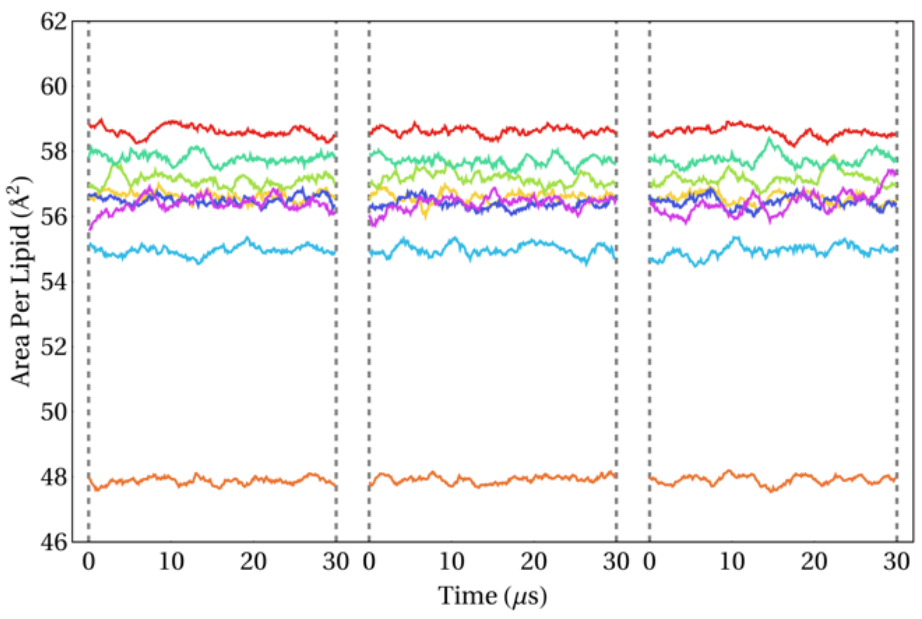

Figure S3. Area per lipid over time for replica 1 (left), 2 (middle) and 3 (right) in each of the 8 membranes modelled. Timeseries were smoothed using a running average filter. See Figure 1 and Table S1 for composition of each membrane. 
a)

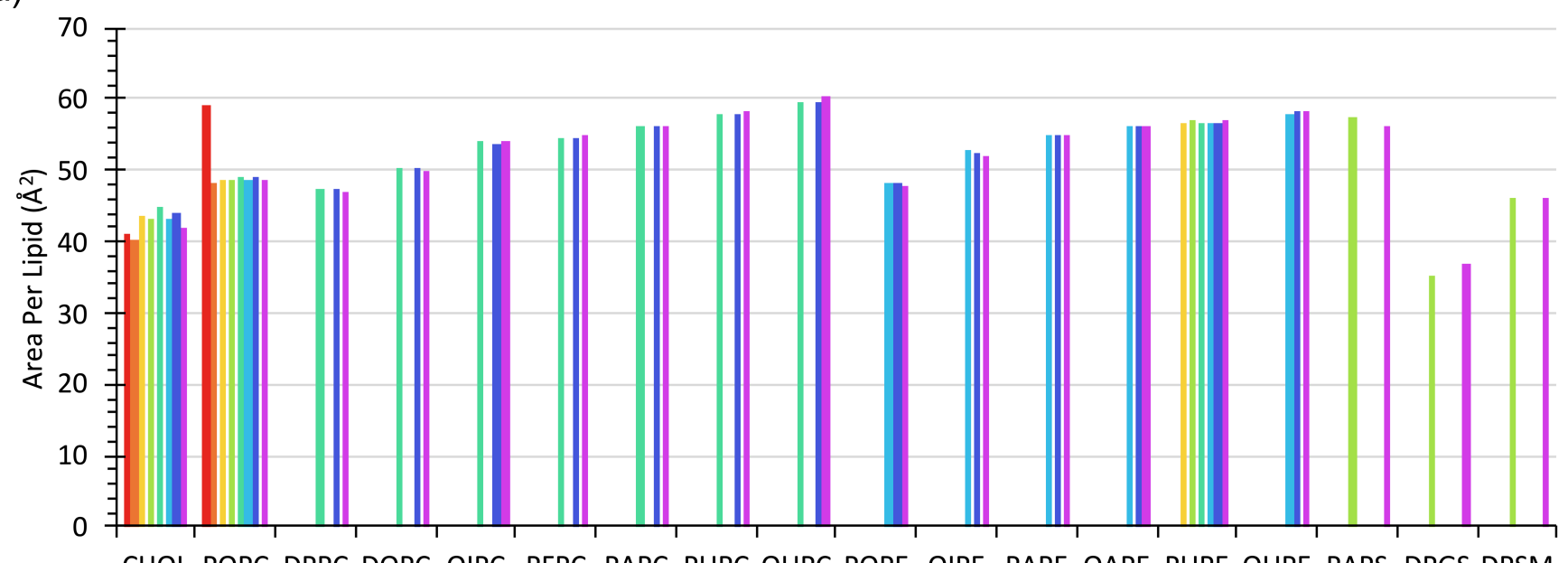

CHOL POPC DPPC DOPC OIPC PFPC PAPC PUPC OUPC POPE OIPE PAPE OAPE PUPE OUPE PAPS DPGS DPSM

b)

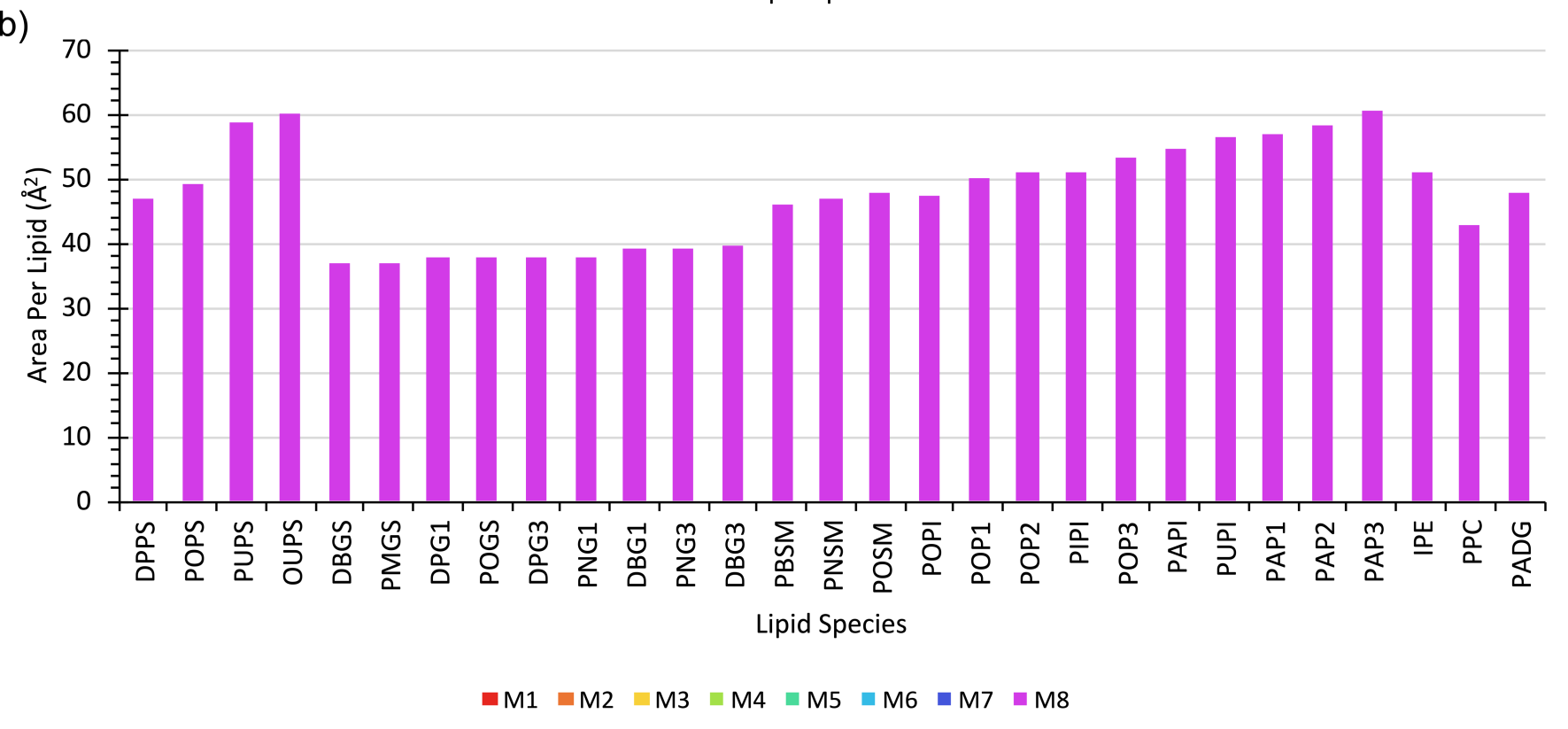

Figure S4. Average area per lipid for each of the a) unique lipid species in the 8 membranes or b) additional species only found in the neuronal membrane modelled over the full $90 \mu$ s of simulation. See Figure 1 and Table S1 for composition of each membrane. 

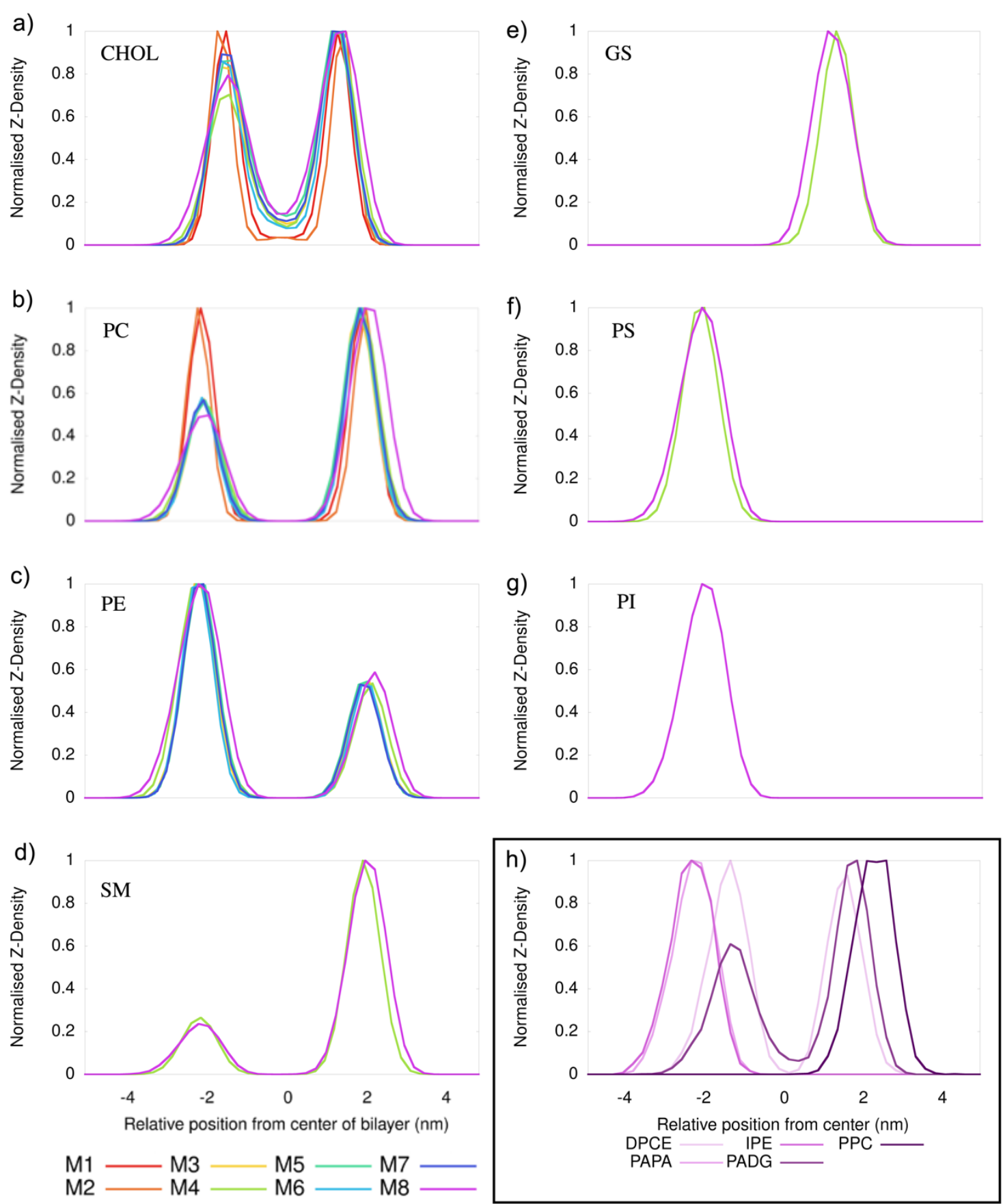

Figure S5. Normalized Z-density of the a) cholesterol, b) PC, c) PE, d) SM, e) GS, f) PS, g) PI, and h) other headgroups in the 8 membranes modelled over the combined $90 \mu$ s of simulation. See Figure 1 and Table S1 for composition of each membrane. 


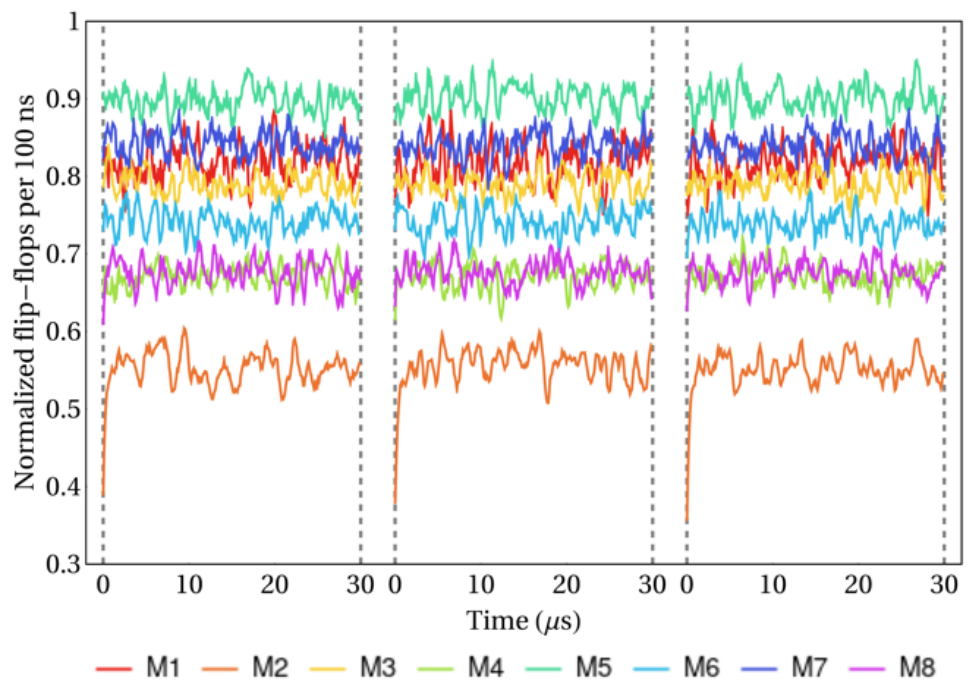

Figure S6. Cholesterol flipping rate over time for replica 1 (left), 2 (middle) and 3 (right) in each of the 8 membranes modelled. Timeseries were smoothed using a running average filter. See Figure 1 and Table S1 for composition of each membrane. 

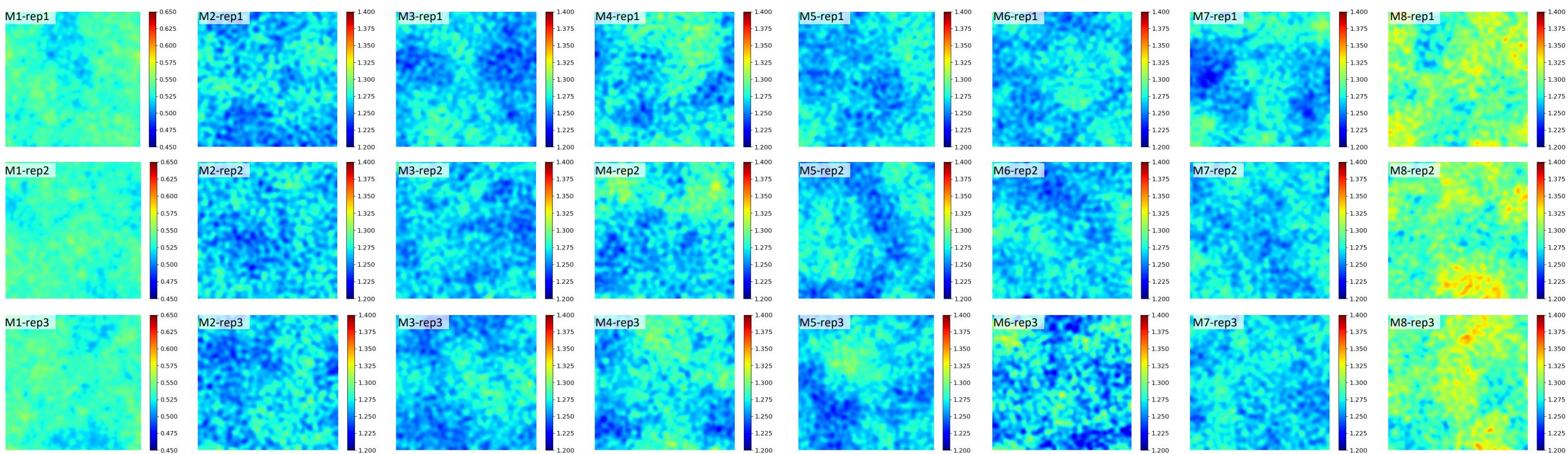

Figure S7. Average lateral density (molecules $/ \mathrm{nm}^{3}$ ) of cholesterol over each $30 \mu$ r replicate for the 8 different membrane compositions modelled. See Figure 1 and Table S1 for composition of each membrane. 


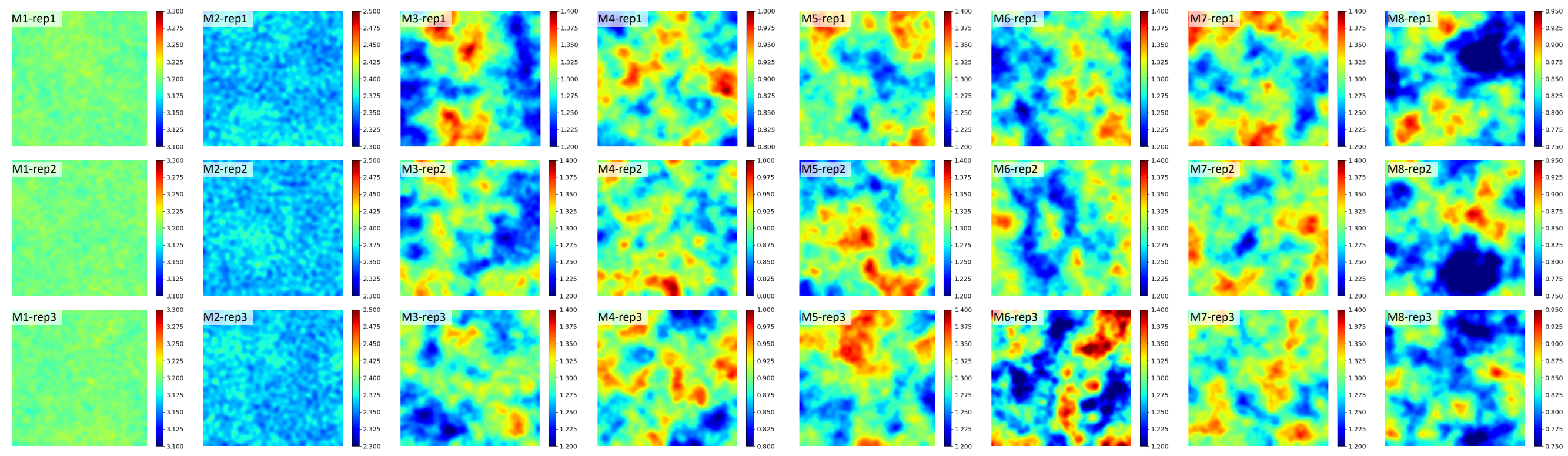

Figure S8. Average lateral density (molecules $/ \mathrm{nm}^{3}$ ) of PC lipids over each $30 \mu$ s replicate for the 8 different membrane compositions modelled. See Figure 1 and Table S1 for composition of each membrane. 


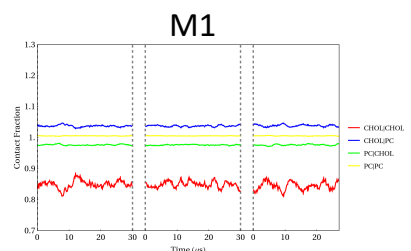

$\mathrm{M} 2$

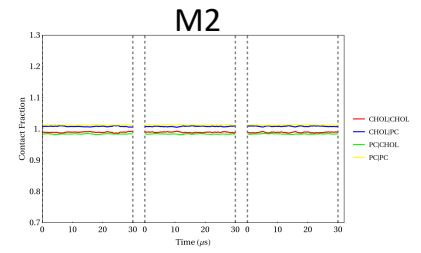

M3

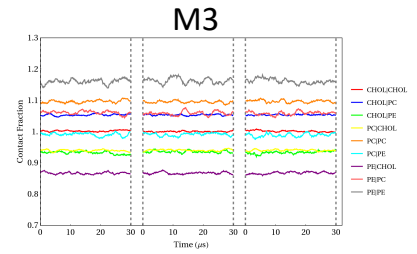

M4

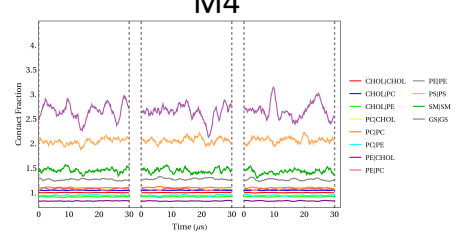

M5

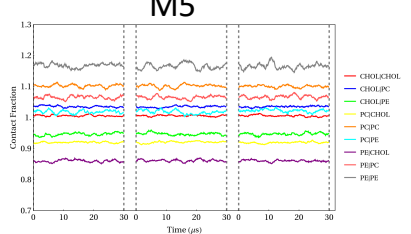

M6

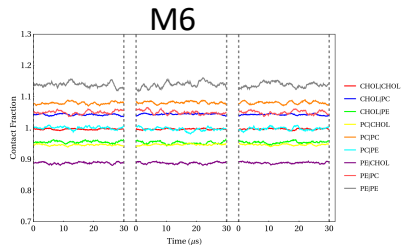

M7

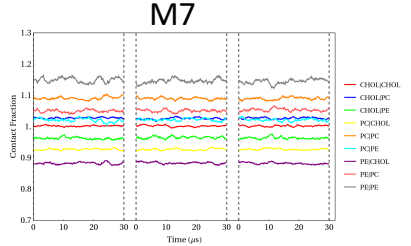

M8

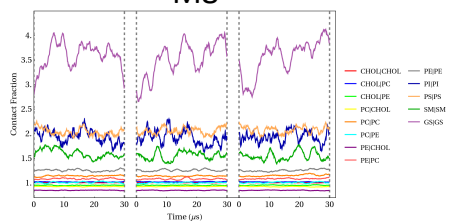

Figure S9. Average lipid contact fractions over time for replica 1 (left), 2 (middle) and 3 (right) for each of the 8 membranes modelled. See Figure 1 and Table S1 for composition of each membrane. 


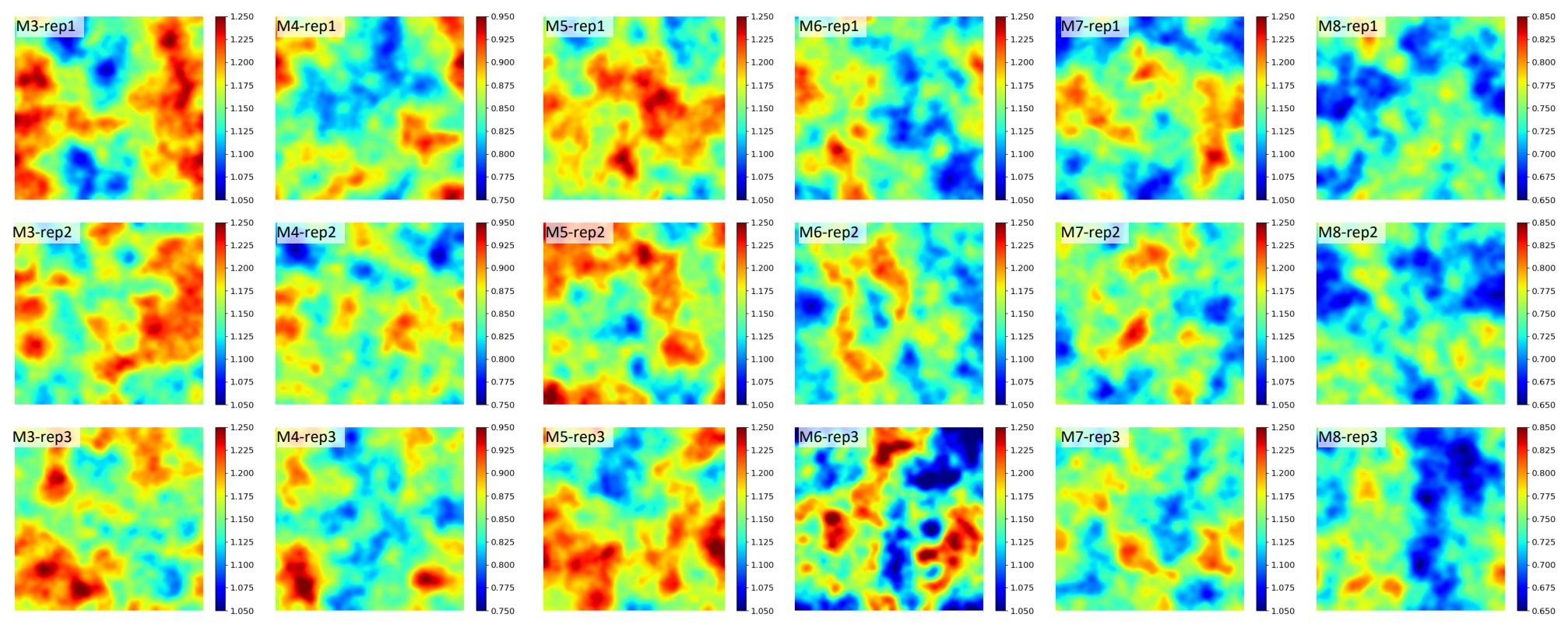

Figure S10. Average lateral density (molecules $/ \mathrm{nm}^{3}$ ) of PE lipids over each $30 \mu$ s replicate for the 8 different membrane compositions modelled. See Figure 1 and Table S1 for composition of each membrane. 

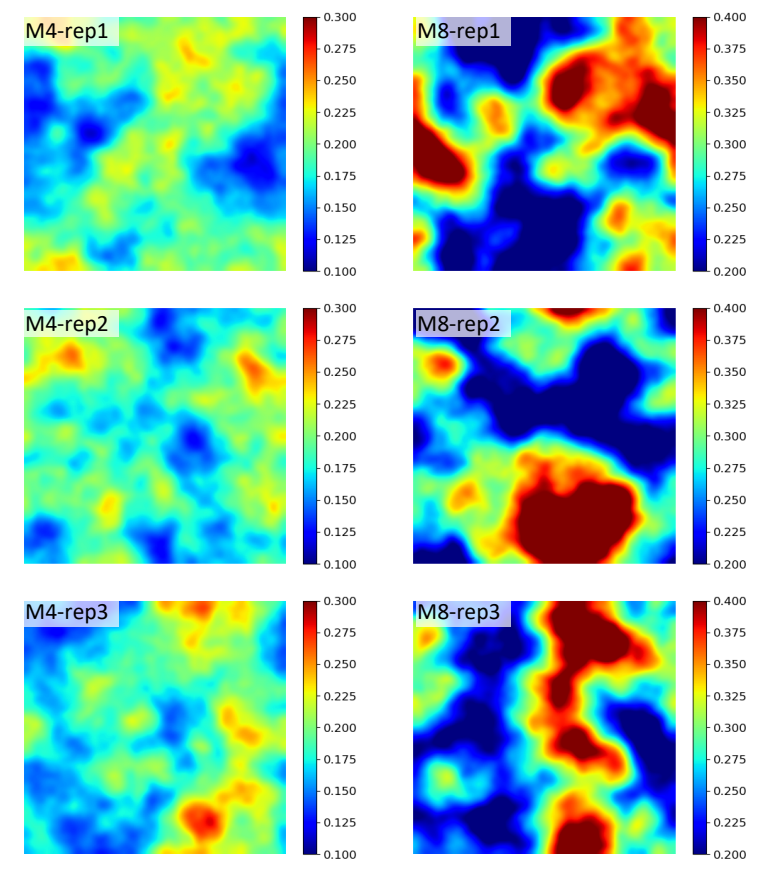

Figure S11. Average lateral density (molecules $/ \mathrm{nm}^{3}$ ) of GS lipids over each $30 \mu$ s replicate for the 8 different membrane compositions modelled. See Figure 1 and Table S1 for composition of each membrane. 

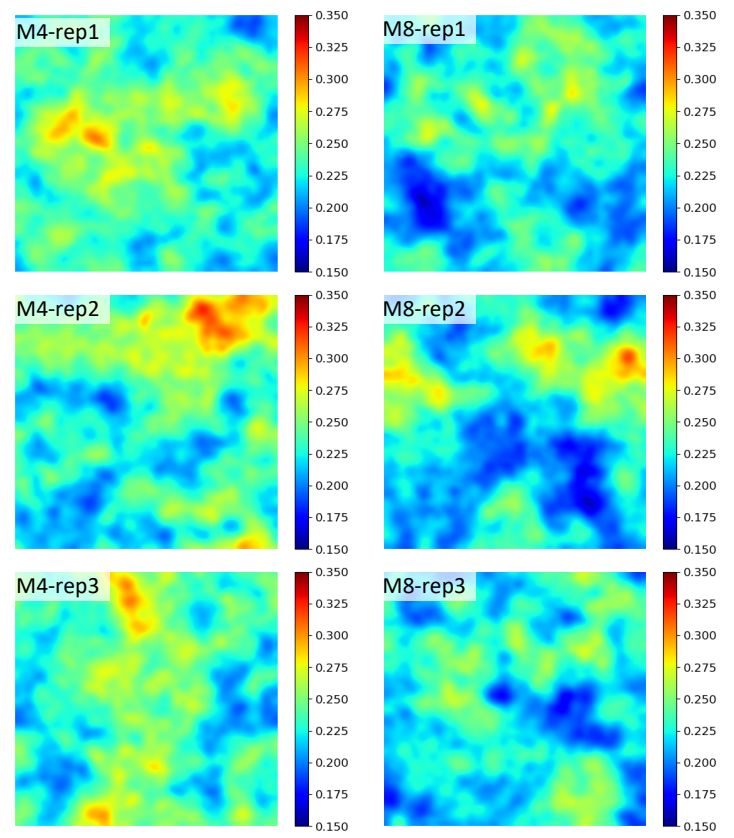

Figure S12. Average lateral density (molecules $/ \mathrm{nm}^{3}$ ) of SM lipids over each $30 \mu$ s replicate for the 8 different membrane compositions modelled. See Figure 1 and Table S1 for composition of each membrane.
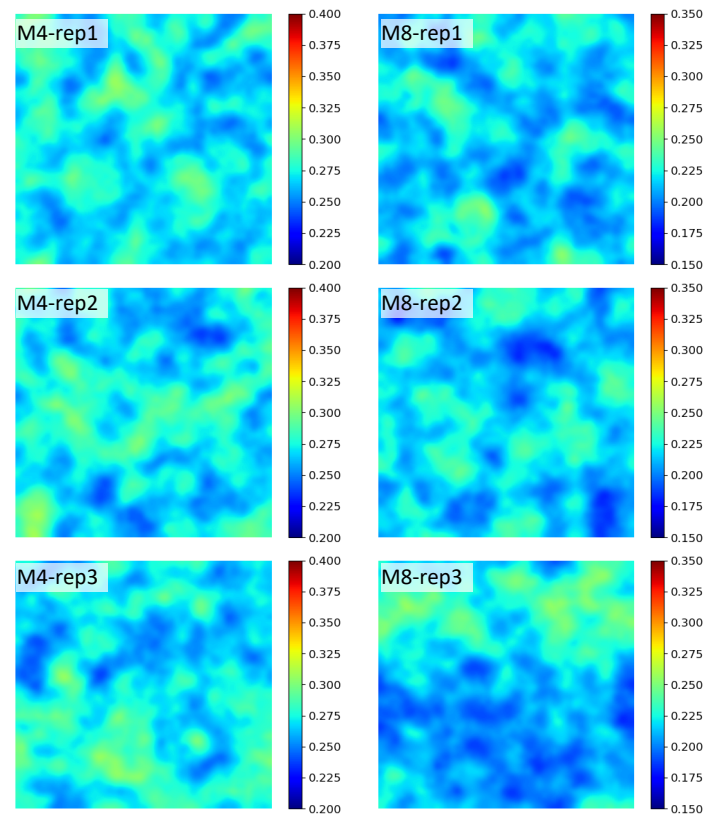

Figure S13. Average lateral density (molecules $/ \mathrm{nm}^{3}$ ) of PS lipids over each $30 \mu$ s replicate for the 8 different membrane compositions modelled. See Figure 1 and Table S1 for composition of each membrane. 

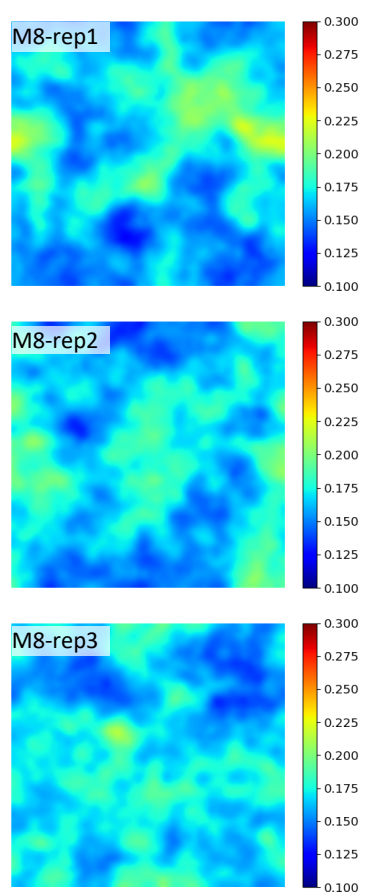

Figure S14. Average lateral density (molecules $/ \mathrm{nm}^{3}$ ) of PI lipids over each $30 \mu$ s replicate for the 8 different membrane compositions modelled. See Figure 1 and Table S1 for composition of each membrane.
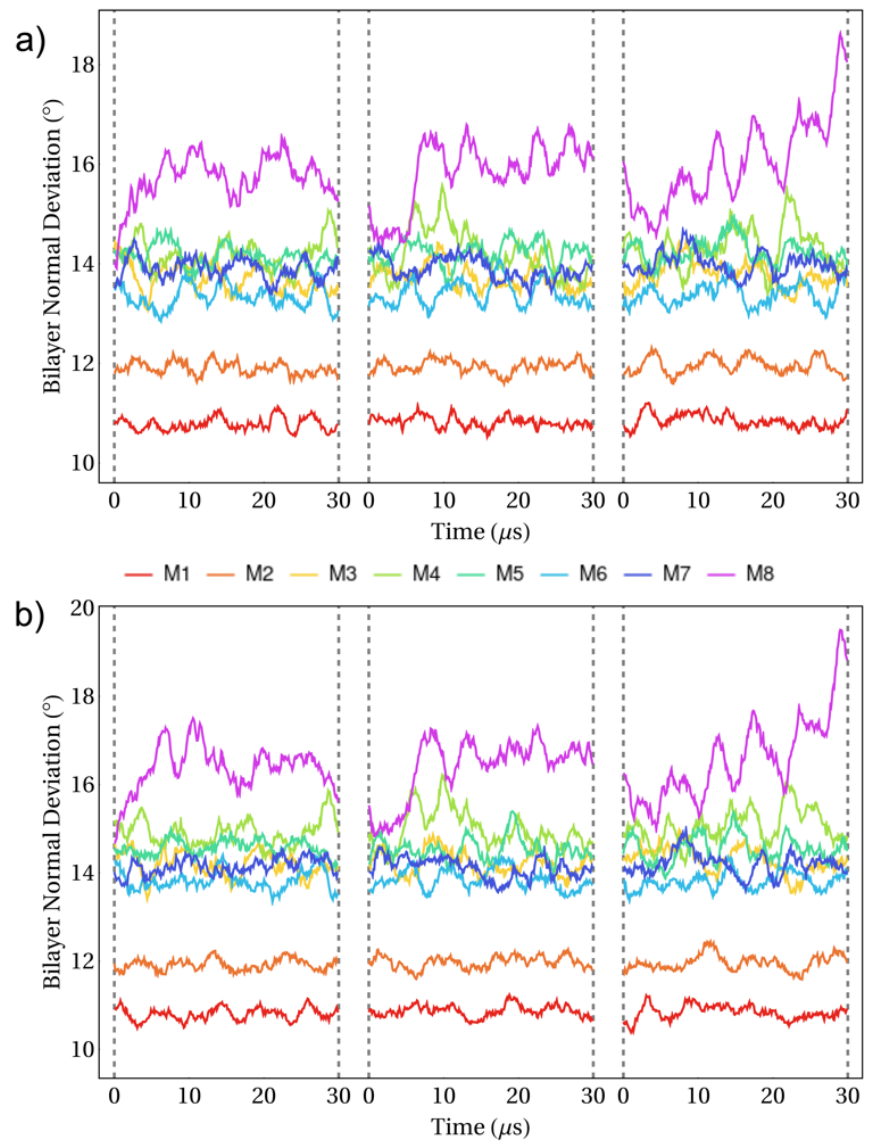

Figure S15. Bilayer normal deviation in the a) extracellular or b) intracellular leaflet over time for replica 1 (left), 2 (middle) and 3 (right) for each of the 8 membranes modelled. Timeseries were smoothed using a running average filter. See Figure 1 and Table S1 for composition of each membrane. 


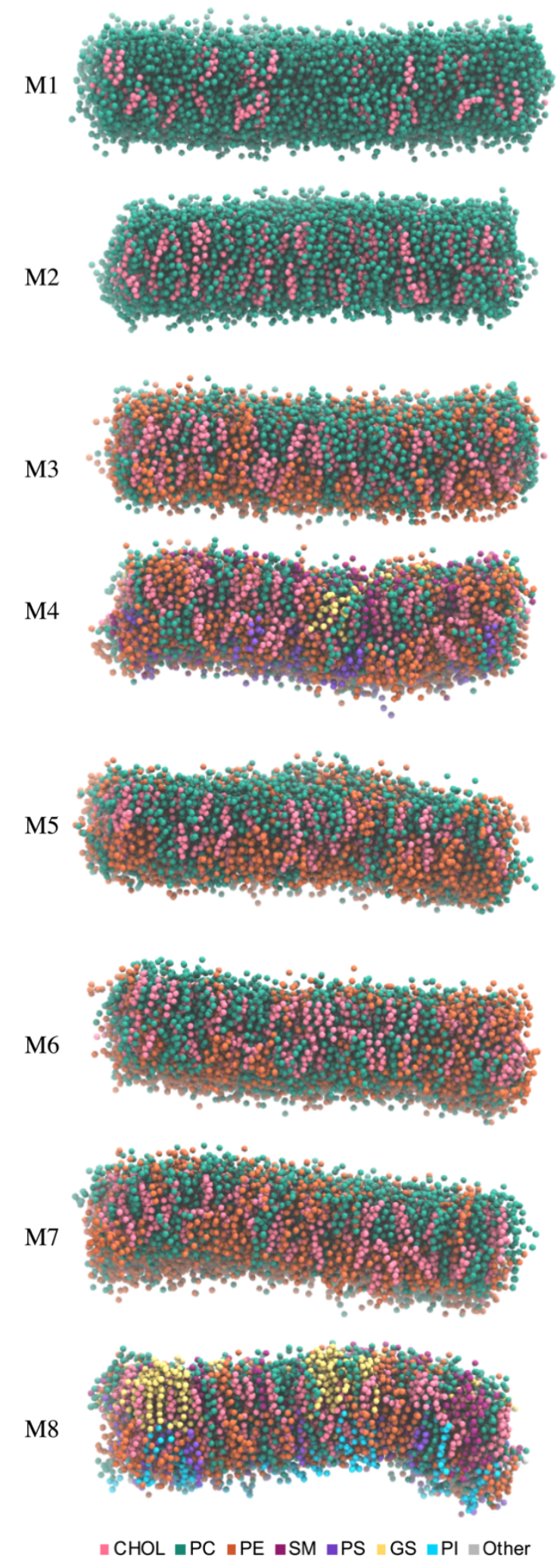

Figure S16. Side view showing the membrane curvature of each of the 8 membranes modelled at the end of a $30 \mu \mathrm{s}$ simulation. See Figure 1 and Table S1 for composition of each membrane. 